\title{
Commercial Dengue Rapid Diagnostic Tests for Point-of-Care Application: Recent Evaluations and Future Needs?
}

\author{
Stuart D. Blacksell ${ }^{1,2}$ \\ ${ }^{1}$ Center for Tropical Medicine, Nuffield Department of Clinical Medicine, Churchill Hospital, University of Oxford, Oxford, UK \\ ${ }^{2}$ Mahidol Oxford Tropical Medicine Research Unit, Faculty of Tropical Medicine, Mahidol University, 420/6 Rajvithi Road, \\ Bangkok, 10400, Thailand
}

Correspondence should be addressed to Stuart D. Blacksell, stuart@tropmedres.ac

Received 12 December 2011; Accepted 11 February 2012

Academic Editor: Roy A. Hall

Copyright (c) 2012 Stuart D. Blacksell. This is an open access article distributed under the Creative Commons Attribution License, which permits unrestricted use, distribution, and reproduction in any medium, provided the original work is properly cited.

\begin{abstract}
Dengue fever, dengue haemorrhagic fever, and dengue shock syndrome (DF/DHF/DSS) are tropical diseases that cause significant humanitarian and economic hardship. It is estimated that more than 2.5 billion people are at risk of infection and more than 100 countries have endemic dengue virus transmission. Laboratory tests are essential to provide an accurate diagnosis of dengue virus infection so that appropriate treatment and patient management may be administered. In many dengue endemic settings, laboratory diagnostic resources are limited and simple rapid diagnostic tests (RDTs) provide opportunities for point-of-care diagnosis. This paper addresses current issues relating to the application of commercial dengue RDTs for the diagnosis of acute dengue virus infection, recent diagnostic evaluations, and identifies future needs.
\end{abstract}

\section{Introduction}

1.1. The Burden of Dengue. Dengue fever, dengue haemorrhagic fever and dengue shock syndrome (DF/DHF/DSS) are a group of tropical disease states that cause significant humanitarian and economic hardship. DF/DHF/DSS are caused by the dengue virus, which belongs to the flavivirus genus of the family Flaviviridae. The flavivirus genus includes approximately 70 viruses of which there are 3 antigenic complexes; the Japanese encephalitis virus, tickborne encephalitis, and the dengue virus complexes [1]. There are four distinct serotypes of dengue virus (i.e., dengue virus serotypes 1-4) which all cause clinical disease. It is estimated that more than 2.5 billion people are at risk of infection and more than 100 countries have endemic dengue virus transmission. While exact numbers of dengue virus cases are not available, for the period 2000-2004, the annual average was 925,896 cases, which was almost double when compared to the 479,848 cases that were reported for the period 1990-1999 [2]. About 250,000 to 500,000 cases of DHF are reported annually although the true incidence is not really known [3]. In dengue endemic regions which include countries in Asia and the Americas, the burden of dengue is approximately 1,300 disability-adjusted life years (DALYs) per million population, which is similar to the disease burden of other childhood and tropical diseases, including tuberculosis, in these regions [2].

1.2. Why Do We Need Rapid Diagnostic Tests (RDTs) and Who Controls the Quality? Laboratory tests are essential to provide an accurate diagnosis of acute dengue virus infection at patient presentation to a clinical setting so that appropriate treatment and patient management may be administered. In many dengue endemic settings, laboratory diagnostic resources are limited and simple rapid diagnostic tests (RDTs) provide opportunities for point-of-care diagnosis. The characteristics of the ideal diagnostic test are said to be defined by the ASSURED criteria: (1) Affordable by those at risk of infection; (2) Sensitive (few false-negatives); (3) Specific (few false-positives); (4) User-friendly (simple to perform and requiring minimal training); (5) Rapid (to enable treatment at first visit) and Robust (does not require refrigerated storage); (6) Equipment-free; (7) Delivered to those who need it [4]. 
The need for simple point-of-care diagnostic tests has led to the proliferation of antibody-based RDTs for tropical infections such as dengue, leptospirosis, melioidosis, and malaria using the immunochromatographic test (ICT) format. Unfortunately, many dengue antibody-based RDTs had substandard performance for the diagnosis of acute dengue at patient presentation which leads to the large-scale evaluations funded by independent international organisations such as World Health Organization (WHO) [5, 6] to determine which are the best of the commercial assays. Until these large-scale evaluations were performed, many "backyard" manufacturers marketed their products via the internet with little or no independent verification of the manufacturer's performance claims. Results from these evaluations have provided independent performance details to consumers, and poor results challenged manufacturers to improve RDT performance. The RDT market still remains largely unregulated with the exception of the USA where in vitro devices require approval by the Food and Drug Administration (FDA) however, in the absence of national regulations, high-quality, independent assessments in peerreviewed journals provide the best guide to quality.

1.3. Rapid Test Formats. Immunochromatographic tests for the detection of dengue virus nonstructural protein 1 (NS1) antigen, IgM, IgG, and IgA antibodies have been developed by a number of commercial companies and have found wide application because of their ease of use and rapidity of results. These dengue RDTs are presented in the form of a lateral flow cassette that allows the flow of sample in a horizontal plane or a wick-style test that is performed in a tube and draws sample vertically by capillary action. Dengue virus RDTs use a cocktail of dried antigens and colloidal gold-labelled monoclonal antibodies (specific for dengue NS1 antigen, IgM, IgG, or IgA antibodies) on a pad at the head of a nitrocellulose strip which is impregnated with either antidengue NS1 antigen, IgM, IgG, or IgA antibody lines. Test sample and running buffer are added to the pad which releases the colloidal gold from the pad and facilitates the mixing of the patient sample with the gold complex and facilitates the migration of the reagents and sample by capillary action along the nitrocellulose strip towards the anti-human IgM, IgG, or IgA antibody lines. The presence of dengue virus NS1 antigen or IgM, IgG, or IgA antibodies is signified by the development of maroon lines in the location of the antibody lines. The dengue RDTs have the advantage that they can be performed in approximately 10-15 minutes and requires no specialized equipment or training, making them ideal for low-technology environments; however, this format has the weakness of subjective reading by the operator.

1.4. Rapid Test Evaluation Methodologies. Diagnostic assays are usually evaluated in terms of sensitivity and specificity that is calculated using a $2 \times 2$ cross-tabulation where a "gold standard" result (the peer-acknowledged, most accurate test) or reference standard result (normally, the test most widely used) is compared with the rapid test to determine diagnostic accuracy. A test that is $100 \%$ sensitive and specific is deemed to be a perfect test. The choice of gold standard assay, final patient result, or comparison with nonreference assay as the reference comparator can have a large influence on the final diagnostic accuracy results. Unfortunately, there is a lack of conformity in the evaluation methodologies and choice of reference assays for dengue RDT diagnostic assessments; however, it should be noted that this issue is not confined only to dengue diagnostics. Guidelines for the evaluations of dengue diagnostic assays have recently been published [7] which is hoped will provide a framework for a uniform approach to diagnostic assessments.

\subsection{Dynamics of Dengue Virus Infection: Implications for} Diagnosis. The dynamics of dengue virus infection have a potentially large influence on the interpretation of RDTs (Figures 1 and 2). Following the initial infection, the dengue virus replicates to high titers in the blood before patients are unwell enough to present to a physician, with viraemia peaking at the time or shortly after the onset of symptoms. Virus remains detectable in the blood for up to 2 to 12 days after the onset of symptoms and may reach titers of up to $1 \times 10^{8} 50 \%$ infectious doses $\left(\mathrm{ID}_{50}\right)$ per $\mathrm{mL}$ or $10^{8.5} 50 \%$ mosquito infectious doses $\left(\mathrm{MID}_{50}\right)[8,9]$. During the viraemic phase of dengue infection, NS1 antigen is produced concomitantly during the virus replication process. NS1 antigen is a 46- to 50-kilodalton glycoprotein highly conserved by all dengue serotypes and is expressed in either membrane-associated or secreted forms $[10,11]$. Soluble NS1 circulates in the serum of patients during the viraemic phase of infection of dengue virus infections and hence is an excellent diagnostic target for acute dengue diagnosis. Difference in the persistence of soluble NS1 antigen in serum between primary (5-6 days post-onset of illness) and secondary dengue infections ( $6-12$ days) has been, noted and it is hypothesised that the presence of anti-NS1 antibodies, that are more frequently detected in dengue secondary infection [12], modulates the formation of antibody-antigen complexes which impede the ability of the test to detect free NS1 antigen [13, 14].

An understanding of the features of the host humoral immune response to dengue virus infection also is important for the interpretation of dengue RDTs. Dengue IgM antibodies are a reliable marker of recent infection but not necessarily acute infection. In primary dengue virus infections, IgM antibodies develop following the decline of viraemia between days $3-5$ after the onset of infection using very sensitive detection methods $[15,16]$ and reache peak levels approximately 2 weeks later [17]. Persistence of IgM antibodies following primary infection using linear regression method has been estimated at 179 days (95\% confidence interval, 155 to 215 days) [18]. In dengue endemic settings where in secondary infections dominate, IgM antibodies may be detectable by RDTs as soon as after 2-3 days of infection [19-21] and peak IgM antibody levels are usually lower than in primary infections $[8,22]$. Persistence of IgM antibodies following secondary infection is estimated to be shorter than that of primary infections at 139 days (95\% confidence interval, 119 to 167 days) [18], and other published estimates of IgM antibody persistence range from 2 months to 6 


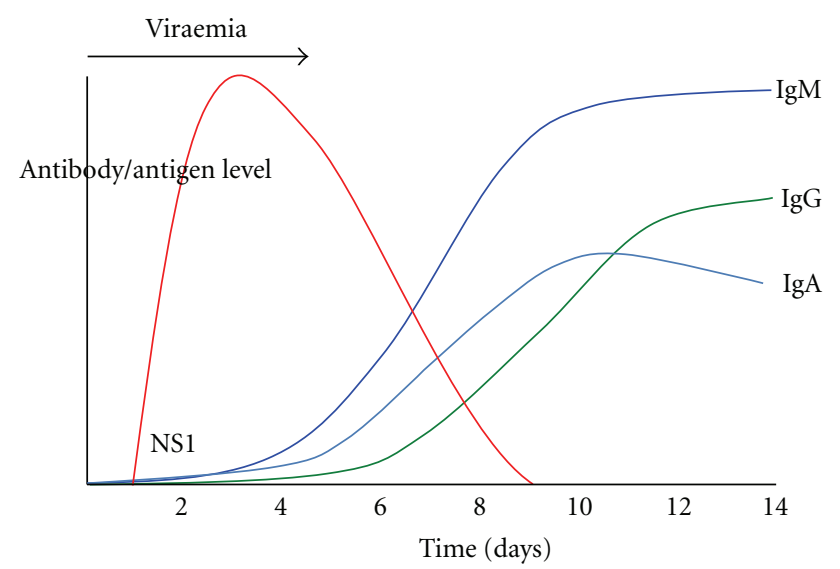

Figure 1: Graphical representation of the kinetics of dengue NS1 antigen and IgM, IgG, and IgA antibodies during a primary dengue infection.

months $[8,23]$. The IgG antibody response develops a few days after the onset of the $\operatorname{IgM}$ antibody response and is serotype specific and may persist for many years following a single infection. Secondary dengue virus infections generate an anamnestic IgG antibody response that is characterised by a rapid rise in IgG antibodies detectable at days 4-5 of illness [16] which is much sooner than the normal IgG antibody response of a primary infection. Dengue IgA antibodies have been reported in serum of dengue fever patients only between days 8 and 11 after onset of fever [17]. However, in the more severe forms of the disease, IgA antibodies were reportedly undetectable in DHF patients in the acute phase of illness (days 2 to 4 ) but increased in the following early convalescent phase (days 5 to 14) and, in DSS patients, increased to the highest levels on days 8 to 11 and slightly decreased 15 days after onset of fever [17]. In primary dengue infection, the onset of detectable levels of $\operatorname{IgA}$ antibodies has been reported on average at 5.5 days after onset of fever, and, in secondary infection, IgA antibodies increased slowly during the first days of the study [22]. The rates of positivity for IgA antibodies in serum were reportedly significantly higher in secondary infections than in primary infections (100 versus $84.6 \%$ ) [24].

\section{Diagnostic Evaluations}

2.1. Performance of Antibody-Based Diagnostics. Dengue IgM and IgG antibody-based RDTs have been in existence for approximately 15 years in various forms by different manufacturers (see Table 1 for description of contemporary commercial dengue IgM and IgG-based RDTs). Multiple diagnostic evaluations were performed from the late 1990s to the mid-2000s [25-29]; however, significant heterogeneity in evaluation methodologies makes direct comparison of diagnostic accuracy problematic [30]. In 2005, WHO Western Pacific Regional Office (WPRO) commissioned an independent evaluation of dengue IgM RDT performance for acute diagnosis as well as an evaluation of storage conditions using stored samples from Thailand [6] and prospectively

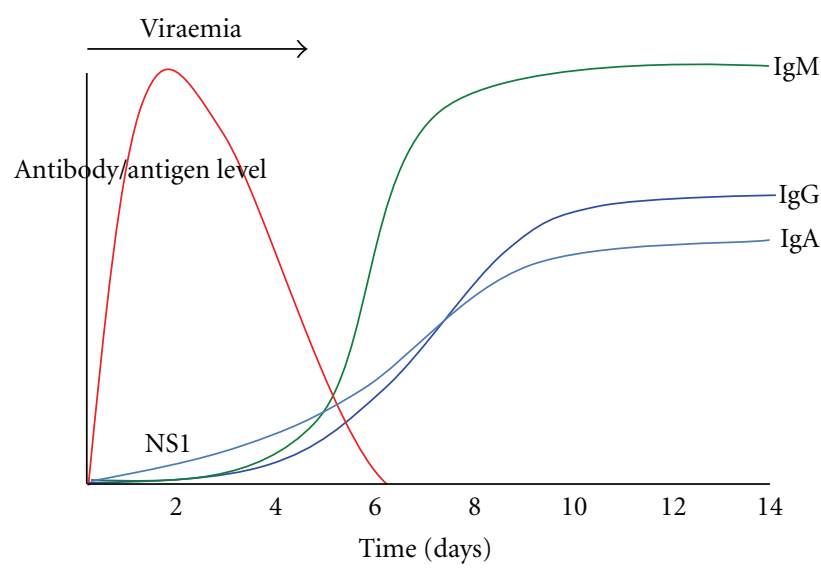

FIGURE 2: Graphical representation of the kinetics of dengue NS1 antigen and IgM, IgG, and IgA antibodies during a secondary dengue infection.

recruited patient samples from Lao PDR [31]. The results for the majority of the evaluated dengue $\operatorname{IgM}$ antibody RDTs demonstrated a lack of sensitivity for acute dengue infection diagnosis that ranged from $6.4 \%$ to $65.3 \%$ and specificities ranged from $69.1 \%$ to $100 \%$ (selected results are presented in Table 2). Subsequently, WHO sponsored a multicentre evaluation of dengue IgM antibody RDTs where test sensitivities ranged from $21 \%$ to $99 \%$ and specificities ranged from $77 \%$ to $98 \%$ when compared with reference ELISAs [5]. Subsequent evaluations of the Panbio Duo IgM RDT reported sensitivities ranging from 65.3 to $81.8 \%$ and specificities ranging from 75.0 to $97.6 \%$ (Table 2). Recent assessments of the Standard Diagnostics (SD) IgM RDT demonstrated improved sensitivity compared to the very poor 1st generation device results from the WHO study [6] $(21.8 \%)$, with 2 nd generation device having reported sensitivities of 53.5\% [21] and 79.2\% [19]. The improvement in the 2nd generation SD IgM RDT is evidence of the positive feedback of diagnostic evaluations to the manufacturers.

2.2. Performance of NS1 Antigen-Based Diagnostics. The most important development in dengue diagnostics in recent years is the advent of the specific detection of dengue virus NS1 antigen (see Table 1 for description of contemporary commercial dengue NS1 antigen RDTs). Dengue RDTs that detect NS1 antigen employ a number of serotype-specific anti-NS1 monoclonal antibodies to capture and detect soluble NS1 antigen in serum, plasma, or blood. The first commercial assays for dengue NS1 antigen detection used the ELISA format $[14,36]$ and demonstrated excellent sensitivity and specificity in the early phase of infection that diminished with falling viraemia levels. The major commercial diagnostics manufacturers, Panbio, Biorad, and SD, have all developed RDT-based NS1 antigen tests, and all have equivalent ELISA-based assays. The diagnostic performance of NS1-based RDTs from the abovementioned manufacturers has been evaluated in numerous geographical locations with the results from 21 diagnostic evaluations presented in Table 3. Twelve studies evaluated the Biorad STRIP RDT 


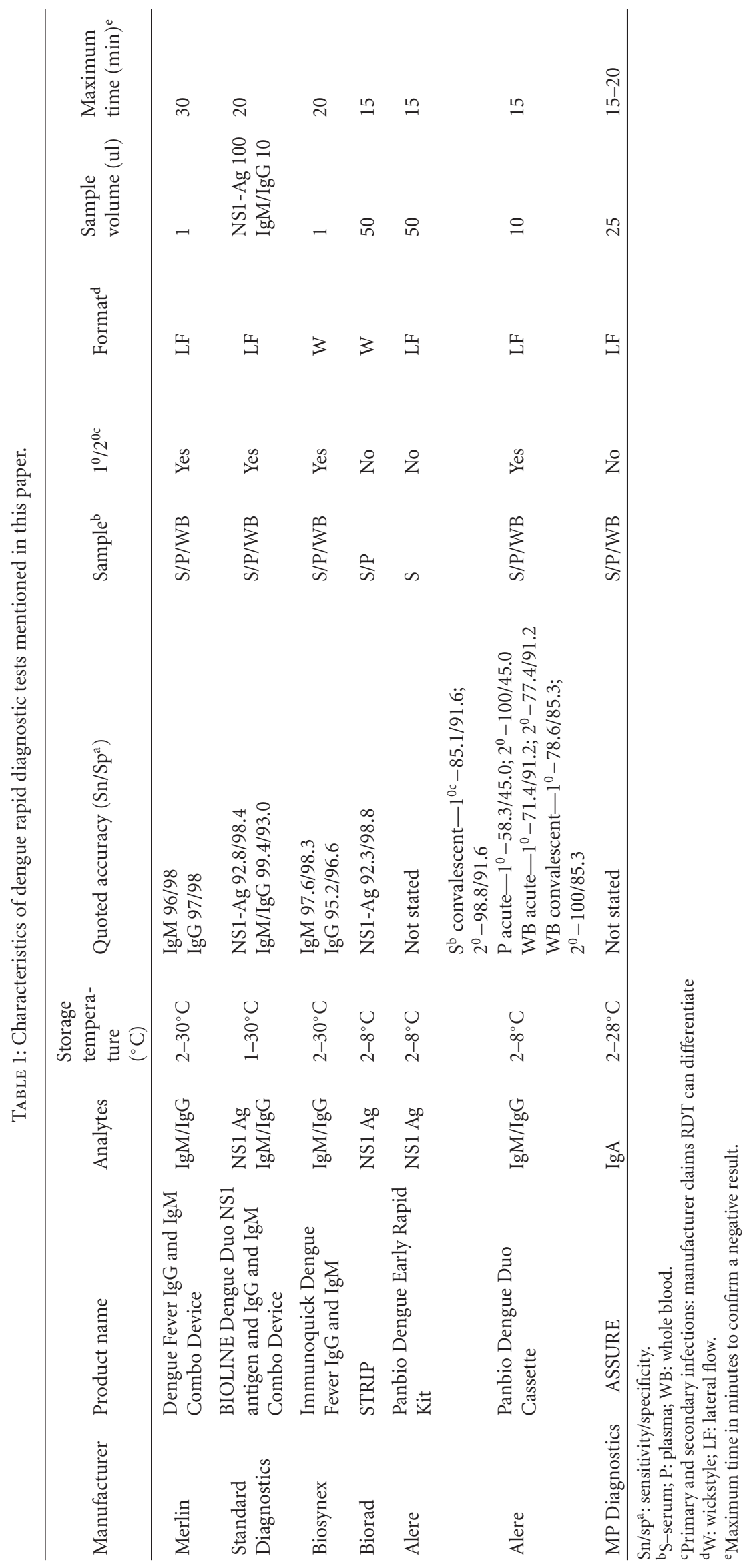




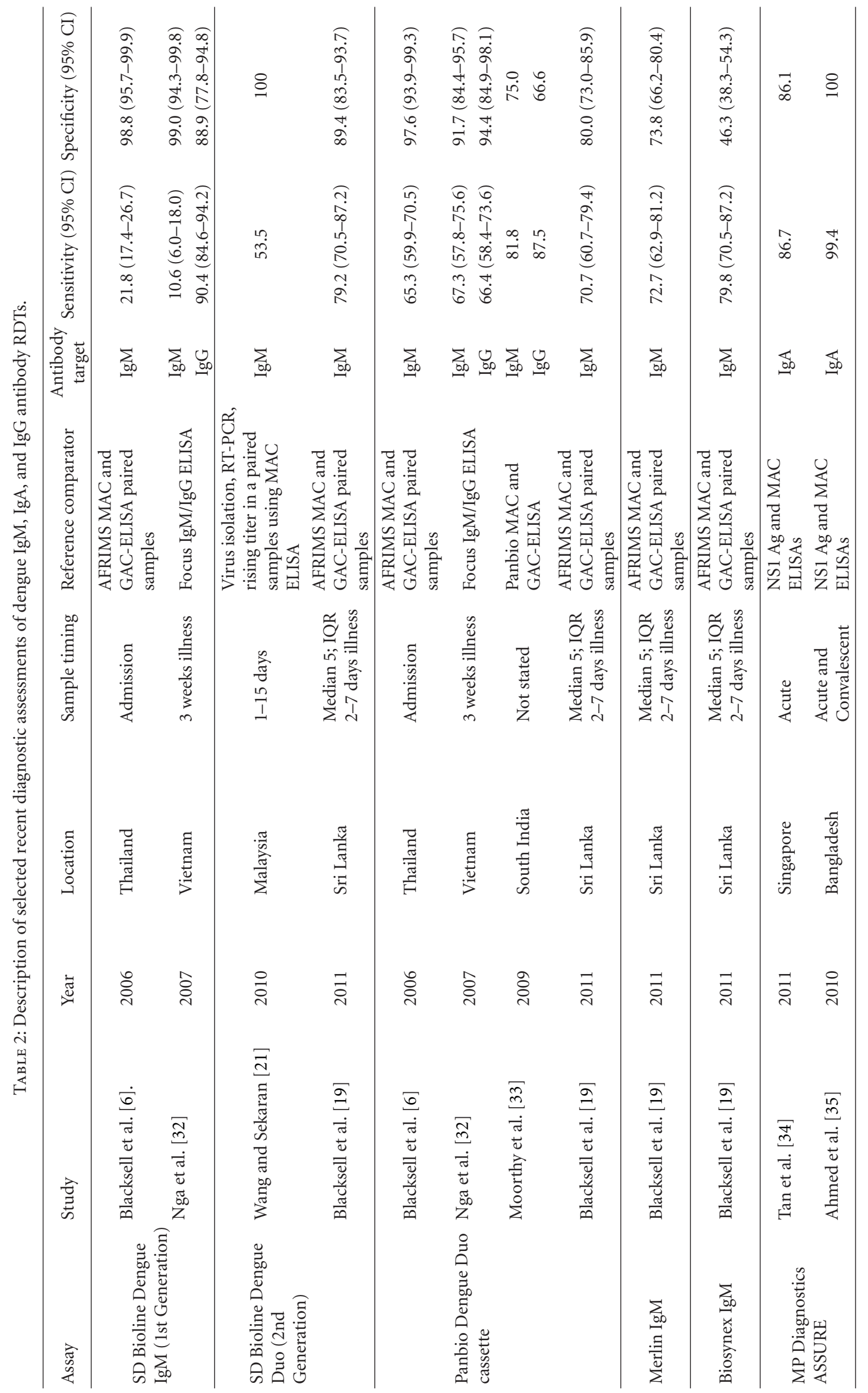


for the diagnosis of acute dengue infection using admission samples, and the results demonstrated considerable variation in sensitivity $(49.8 \%-98.7 \%)$ but the specificities reported were more consistent with all being $>90 \%$. For $25 \%(3 / 12)$ of the studies, the sensitivity was $>89 \%$; however, all of these studies used a skewed comparator of either virus isolation, RT-PCR, or NS1-ELISA and did not examine the possibility of false-negative results by testing paired serum samples to examine for dynamic rise in serological assays such as IgM (MAC) or IgG (GAC) capture ELISAs. Studies that used a more representative combination of virus or antigen detection and serology as reference comparators gave sensitivities for the Biorad STRIP RDT of between 49.4\% [37] and 78.9\% [38]. The SD Bioline Dengue Duo RDT NS1 antigen detection strip was evaluated for acute dengue diagnosis in four studies (Table 3) with consistently high specificity estimates $(96.7-100 \%)$ and sensitivities that ranged from $48.5 \%$ [19] to $65.4 \%$ [21] with the studies either using a combination of virus detection and serology [21, 39, 40] as comparators or serology alone [19]. The Panbio Early Rapid RDT NS1 antigen detection strip was evaluated in two studies using samples from three locations (Vietnam, Malaysia, and Sri Lanka) with high specificity estimates (92.5-96.7\%) and sensitivities that ranged from 58.6\% [19] to $69.2 \%$ [20] for admission samples. A few studies have compared the diagnostic accuracy of NS1 antigen RDTs in primary and secondary dengue infections. Generally, NS1antigen RDTs demonstrated higher sensitivities in primary infections when compared to secondary infections [39, 4143]; however, other studies have reported the opposite [37]. As mentioned earlier, it has been suggested that this phenomenon of lowered NS1-antigen detection in dengue secondary infections is caused by NS1 antigen complexing with anti-NS1 antibodies [12-14]. This observation results in an inability of the NS1-antigen RDT to detect complexed NS1 antigen and should not be interpreted insensitivity on the part of the diagnostic assay.

2.3. Combination of NS1 Antigen and IgM Antibody Results. To take advantage of the entire temporal spectrum of patient presentation during the acute phase of dengue infection (usually from 1 to 7 days after onset of fever), NS1 antigen and $\operatorname{IgM}$ antibody results have been combined in a Boolean manner using AND/OR operators. NS1 antigen is present in the serum in the early phase of infection; however, patients that present late in the course of infection may have undetectable levels of NS1 antigen. Dengue IgM antibodies are usually present following 2-5 days of infection, and, by combining the results of dengue NS1 antigen and IgM antibody testing, accurate diagnosis during acute presentation is afforded. This approach was initially described [48] by combining the results of the Panbio NS1 antigen and IgM antibody ELISAs in Lao PDR. Subsequently, studies [19-21] have combined NS1 antigen and antibody results to exploit the temporal diagnostic characteristics of each analyte (Table 4). Combining the SD Bioline Dengue Duo RDT NS1 antigen and IgM antibody results for acute diagnosis, the sensitivity ranged from $75.5 \%$ [39] to $92.9 \%$ [19] and the specificity from $88.8 \%$ [19] to $100 \%$ [39].
Combining the Panbio Early Rapid RDT NS1 antigen and IgM antibody results, the sensitivity ranged from $89.0 \%$ to $89.9 \%$; the only specificity reported was $75.0 \%$ [19].

\section{Future Needs for Dengue Rapid Tests}

Despite recent improvements in the RDTs, there are a number of issues that require further investigation.

3.1. Standardisation of Diagnostic Assessments. The aforementioned lack of conformity in the evaluation of dengue RDTs remains a problem and a standardised approach must be considered when performing diagnostic assessments so that there is comparability between studies. The recently published guidelines for the evaluations of dengue diagnostic assays [7] should be followed whenever possible.

3.2. Determining Geographical Variation and Practical Aspects of Test Use. To further strengthen the current diagnostic accuracy estimates, prospective recruitment studies are required in different dengue-endemic locations where there are variations in dengue infection status (primary versus secondary), days of illness, and prior to presentation. Further studies are also required to examine some of the more practical aspects of dengue RDT performance that includes the influence of operator training, interoperator variation, and ease of use of the assays. Where case-control studies are to be performed using characterised archived samples, consideration should also be given to the appropriateness of the composition of dengue patient (serotypes, days of illness) and non-dengue patient (other dengue-like fevers) cohorts.

\subsection{Differentiation of Primary versus Secondary Dengue} Infections. Patients with secondary or later dengue infections are considered to have an increased risk of the more severe forms of the disease, and therefore the accurate detection of primary and secondary at presentation to a clinical facility may become a promising patient management tool. Some manufacturers of antibody-based RDTs claim their assays are able to differentiate primary and secondary dengue virus infections using the following criteria: (1) acute primary dengue virus infection defined as an IgM-positive and IgG-negative (IgM+/IgG-) result and (2) acute secondary dengue virus infection defined as IgM-positive and IgGpositive $(\mathrm{IgM}+/ \mathrm{IgG}+)$ or IgM-negative and IgG-positive $(\mathrm{IgM}-/ \mathrm{IgG}+)$ results. Examination of the voracity of the manufacturer's claims is limited to a few studies $[6,19$, 31 ] and is often conducted in dengue endemic settings where there a dominance of secondary dengue infections. Such studies have demonstrated that RDTs cannot reliably differentiate the different dengue infection states.

\subsection{Sample Type and the Effect of Anticoagulants and Preser-} vatives. Many manufacturers allow the use of serum, plasma, or whole blood (Table 1) for use in dengue RDTs in both antigen and antibody formats. Interestingly, the Panbio Duo antibody RDTs only permits the use of serum. Unfortunately, there is little quantitative evidence that all sample types 


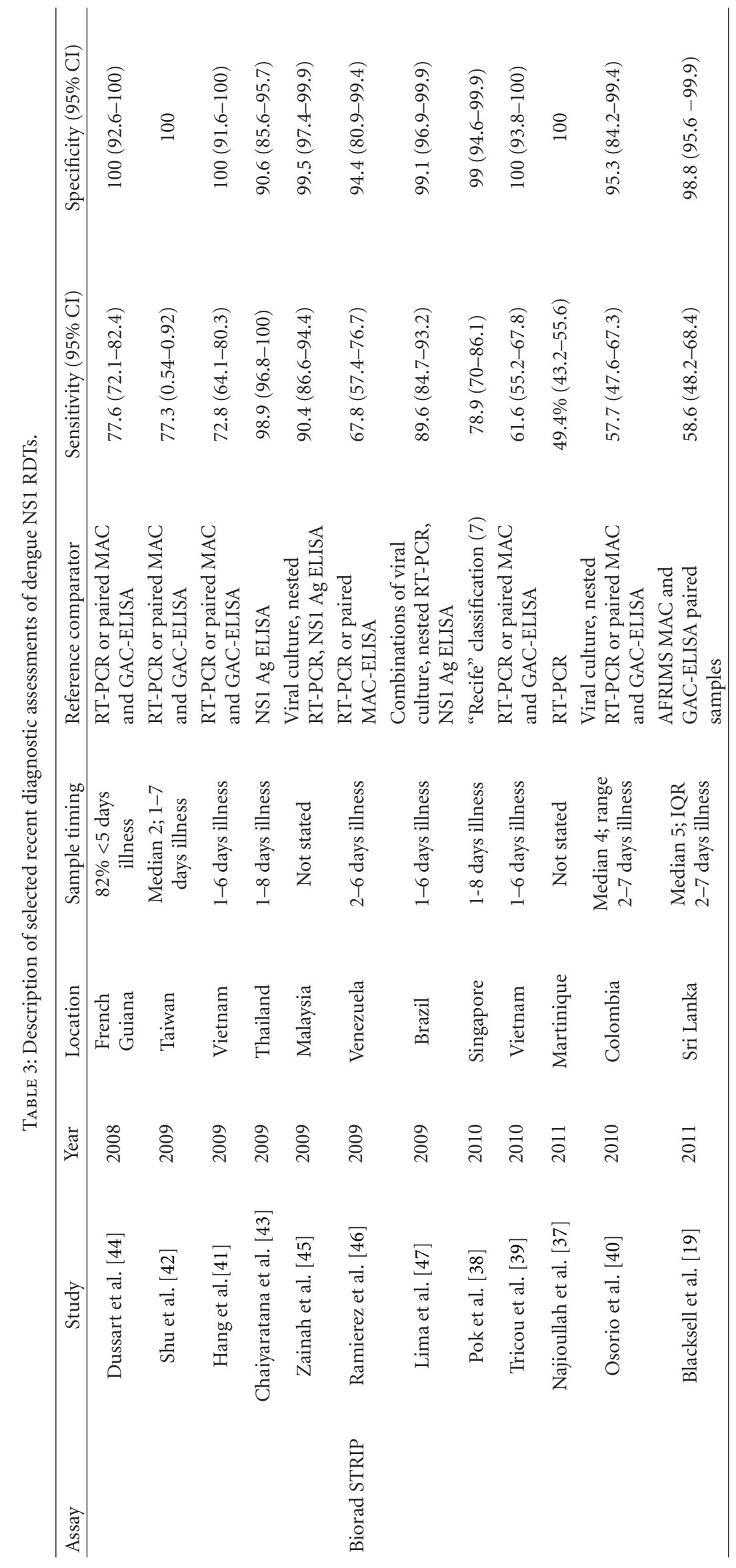




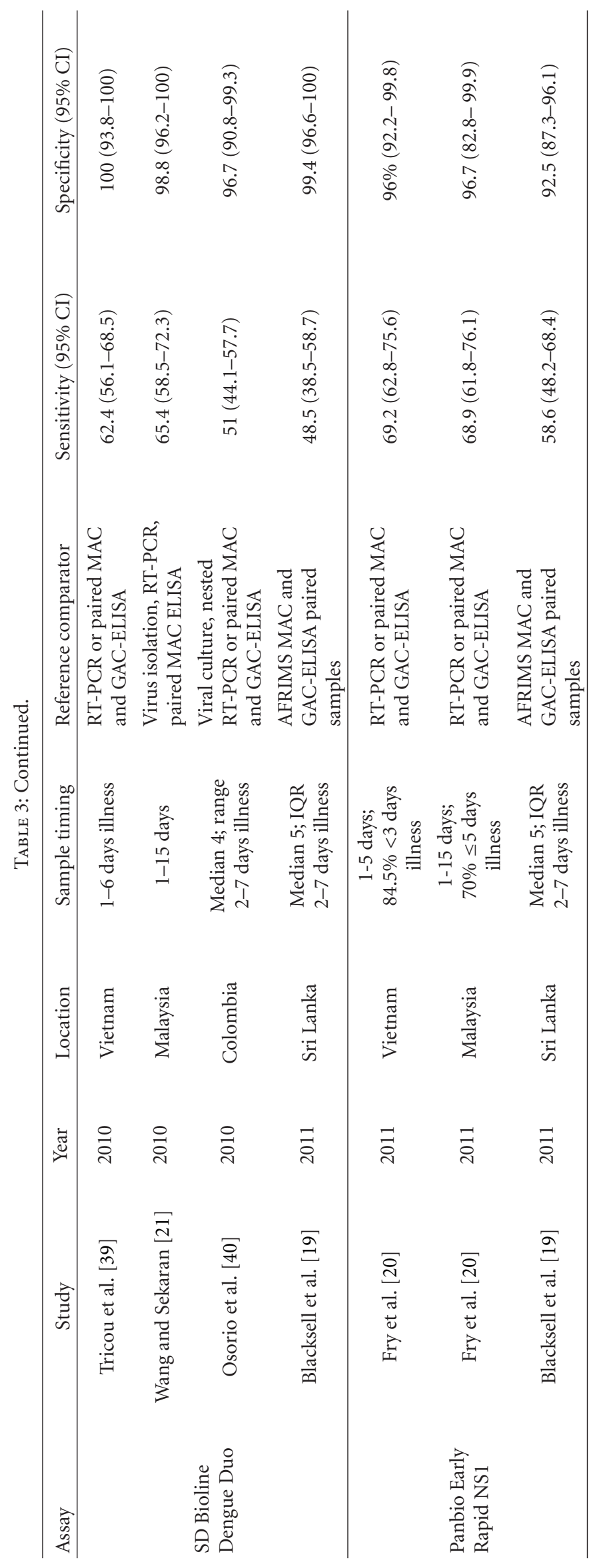




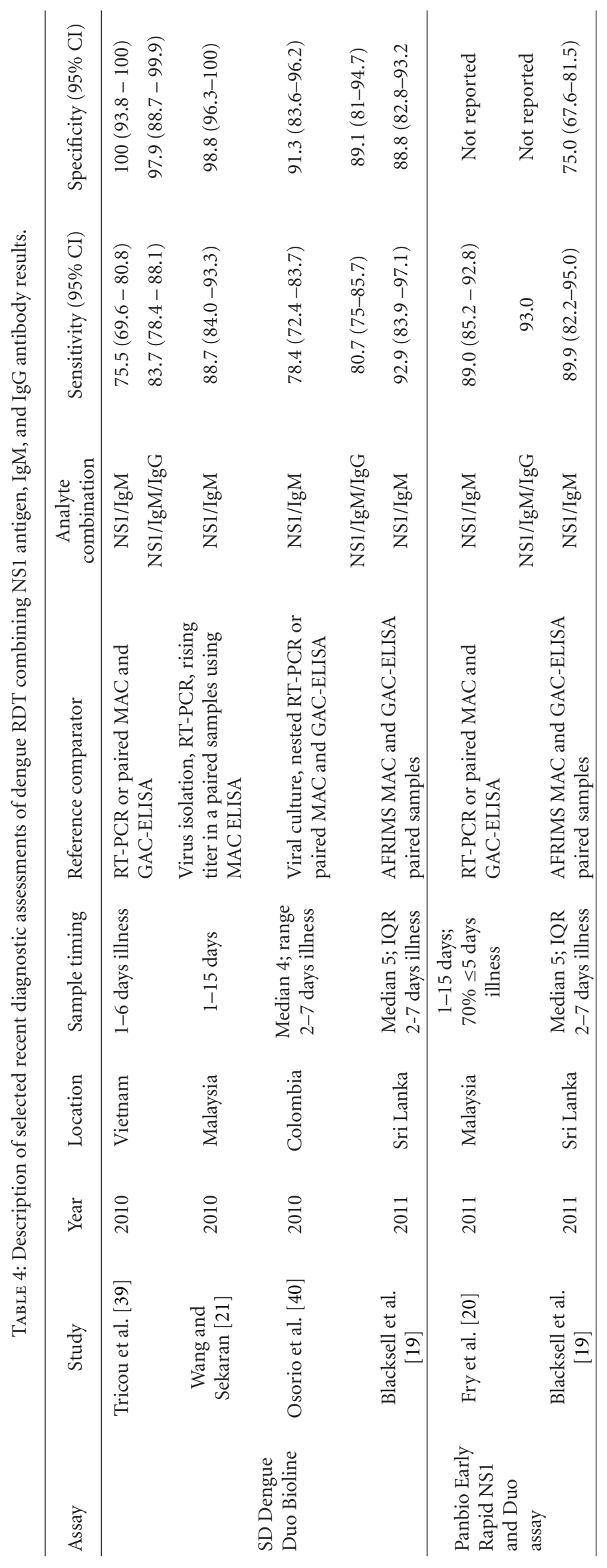


perform equally and it is incumbent of manufacturers to provide these performance details. The effect of sample anticoagulants and whole blood on RDT performance and ease of reading also require examination in a field setting.

3.5. Storage Considerations. Dengue endemic regions are normally located in tropical regions that have high temperature and high humidity climates. Many of the contemporary dengue rapid tests require refrigeration (i.e., $2-8^{\circ} \mathrm{C}$ ) (see Table 1); however, some manufacturers specify storage at 2$30^{\circ} \mathrm{C}$; however, ambient tropical temperatures often exceed $30^{\circ} \mathrm{C}$. There is an urgent need to examine the effect of storage temperature on contemporary dengue RDTs as the only previous investigation concentrated on earlier-generation antibody-based tests [6].

3.6. Prognostic Markers of Disease Severity. While the acute diagnosis of dengue infection is clinically useful, in a dengue endemic setting where the majority of infections are seen in outpatient settings, there is a clear need to also have prognostic details of disease severity. The more severe forms of dengue infection (DHF and DSS) require patient admission to hospital and critical care facilities, and prognostic indicators of clinical severity would provide direction for patient management. Quantifying secreted NS1 antigen has been hypothesised as a marker of disease severity [14], and subsequent studies have shown that dengue NS1 antigen levels correlate with severity disease where plasma levels of secreted NS1 correlated with viraemia levels and were higher in patients with DHF than in those with the clinically less-severe dengue fever [13, 49].

\section{Conclusions}

Despite improvements in the accuracy of IgM-based RDTs, this format is not sufficiently sensitive for acute dengue diagnosis alone. Acute dengue diagnosis using IgG-based RDTs is not recommended due to the lifelong persistence of dengue IgG antibodies and hence the possibility of misdiagnosis by false-positive detection. NS1-antigen-based diagnostics are an important component of modern point of care diagnostics; however, they are only sensitive in the early phase of infection and therefore are not suitable for sole use in dengue-endemic settings where late clinical presentations may occur. To take advantage of the results of testing modalities across the entire temporal spectrum of patient presentation, dengue NS1 antigen, and IgM antibody, RDT results must be combined; however, there is a need to educate clinicians and scientists of this fact. The challenge for manufacturers and researchers is to address the gaps in the more practical aspects of dengue RDT performance including samples types, RDT storage, disease severity, and conduct of future diagnostic assessments.

\section{Acknowledgments}

Stuart D. Blacksell is supported by the Wellcome Trust of Great Britain.

\section{References}

[1] D. J. Gubler, "Dengue and dengue hemorrhagic fever," Clinical Microbiology Reviews, vol. 11, no. 3, pp. 480-496, 1998.

[2] M. G. Guzman, S. B. Halstead, H. Artsob et al., "Dengue: a continuing global threat," Nature Reviews Microbiology, vol. 8, supplement 12, pp. S7-S16, 2010.

[3] M. G. Guzmán and G. Kourí, "Dengue diagnosis, advances and challenges," International Journal of Infectious Diseases, vol. 8, no. 2, pp. 69-80, 2004.

[4] R. W. Peeling and D. Mabey, "Point-of-care tests for diagnosing infections in the developing world," Clinical Microbiology and Infection, vol. 16, no. 8, pp. 1062-1069, 2010.

[5] E. A. Hunsperger, S. Yoksan, P. Buchy et al., "Evaluation of commercially available anti-dengue virus immunoglobulin $\mathrm{M}$ tests," Emerging Infectious Diseases, vol. 15, no. 3, pp. 436-440, 2009.

[6] S. D. Blacksell, P. N. Newton, D. Bell et al., "The comparative accuracy of 8 commercial rapid immunochromatographic assays for the diagnosis of acute dengue virus infection," Clinical Infectious Diseases, vol. 42, no. 8, pp. 1127-1134, 2006.

[7] R. W. Peeling, P. G. Smith, and P. M. M. Bossuyt, "A guide for diagnostic evaluations," Nature Reviews Microbiology, vol. 4, no. 9, pp. S2-S6, 2006.

[8] D. W. Vaughn, S. Green, S. Kalayanarooj et al., "Dengue viremia titer, antibody response pattern, and virus serotype correlate with disease severity," Journal of Infectious Diseases, vol. 181, no. 1, pp. 2-9, 2000.

[9] D. J. Gubler, W. Suharyono, and R. Tan, "Viraemia in patients with naturally acquired dengue infection," Bulletin of the World Health Organization, vol. 59, no. 4, pp. 623-630, 1981.

[10] M. Flamand, F. Megret, M. Mathieu, J. Lepault, F. A. Rey, and V. Deubel, "Dengue virus type 1 nonstructural glycoprotein NS1 is secreted from mammalian cells as a soluble hexamer in a glycosylation-dependent fashion," Journal of Virology, vol. 73, no. 7, pp. 6104-6110, 1999.

[11] A. K. I. Falconar and P. R. Young, "Production of dimerspecific and dengue virus group cross-reactive mouse monoclonal antibodies to the dengue 2 virus non-structural glycoprotein NS1," Journal of General Virology, vol. 72, no. 4, pp. 961-965, 1991.

[12] P. Koraka, C. P. Burghoorn-Maas, A. Falconar et al., "Detection of immune-complex-dissociated nonstructural-1 antigen in patients with acute dengue virus infections," Journal of Clinical Microbiology, vol. 41, no. 9, pp. 4154-4159, 2003.

[13] D. H. Libraty, P. R. Young, D. Pickering et al., "High circulating levels of the dengue virus nonstructural protein NS1 early in dengue illness correlate with the development of dengue hemorrhagic fever," Journal of Infectious Diseases, vol. 186, no. 8, pp. 1165-1168, 2002.

[14] P. R. Young, P. A. Hilditch, C. Bletchly, and W. Halloran, "An antigen capture enzyme-linked immunosorbent assay reveals high levels of the dengue virus protein NS1 in the sera of infected patients," Journal of Clinical Microbiology, vol. 38, no. 3, pp. 1053-1057, 2000.

[15] D. Hu, B. Di, X. Ding et al., "Kinetics of non-structural protein $1, \operatorname{IgM}$ and IgG antibodies in dengue type 1 primary infection," Virology Journal, vol. 8, p. 47, 2011.

[16] A. Sa-Ngasand, S. Anantapreecha, A. A-Nuegoonpipat et al., "Specific IgM and IgG responses in primary and secondary dengue virus infections determined by enzyme-linked immunosorbent assay," Epidemiology and Infection, vol. 134, no. 4 , pp. 820-825, 2006. 
[17] P. Koraka, C. Suharti, T. E. Setiati et al., "Kinetics of dengue virus-specific serum immunoglobulin classes and subclasses correlate with clinical outcome of infection," Journal of Clinical Microbiology, vol. 39, no. 12, pp. 4332-4338, 2001.

[18] H. E. Prince and J. L. Matud, "Estimation of dengue virus IgM persistence using regression analysis," Clinical and Vaccine Immunology, vol. 18, no. 12, pp. 2183-2185, 2011.

[19] S. D. Blacksell, R. G. Jarman, M. S. Bailey et al., "Evaluation of six commercial point-of-care tests for diagnosis of acute dengue infections: the need for combining NS1 antigen and IgM/IgG antibody detection to achieve acceptable levels of accuracy," Clinical and Vaccine Immunology, vol. 18, no. 12, pp. 2095-2101, 2011.

[20] S. R. Fry, M. Meyer, M. G. Semple et al., "The diagnostic sensitivity of Dengue Rapid test assays is significantly enhanced by using a combined Antigen and Antibody testing approach," PLoS Neglected Tropical Diseases, vol. 5, no. 6, p. e1199, 2011.

[21] S. M. Wang and S. D. Sekaran, "Evaluation of a commercial SD dengue virus NS1 antigen capture enzyme-linked immunosorbent assay kit for early diagnosis of dengue virus infection," Journal of Clinical Microbiology, vol. 48, no. 8, pp. 2793-2797, 2010.

[22] S. Vázquez, S. Cabezas, A. B. Pérez et al., "Kinetics of antibodies in sera, saliva, and urine samples from adult patients with primary or secondary dengue 3 virus infections," International Journal of Infectious Diseases, vol. 11, no. 3, pp. 256-262, 2007.

[23] B. L. Innis, A. Nisalak, S. Nimmannitya et al., "An enzymelinked immunosorbent assay to characterize dengue infections where dengue and Japanese encephalitis co-circulate," American Journal of Tropical Medicine and Hygiene, vol. 40, no. 4, pp. 418-427, 1989.

[24] A. Balmaseda, M. G. Guzmán, S. Hammond et al., "Diagnosis of dengue virus infection by detection of specific immunoglobulin M (IgM) and IgA antibodies in serum and saliva," Clinical and Diagnostic Laboratory Immunology, vol. 10, no. 2, pp. 317-322, 2003.

[25] S. J. L. Wu, H. Paxton, B. Hanson et al., "Comparison of two rapid diagnostic assays for detection of immunoglobulin $\mathrm{M}$ antibodies to dengue virus," Clinical and Diagnostic Laboratory Immunology, vol. 7, no. 1, pp. 106-110, 2000.

[26] S. L. Branch and P. N. Levett, "Evaluation of four methods for detection of immunoglobulin M antibodies to dengue virus," Clinical and Diagnostic Laboratory Immunology, vol. 6, no. 4, pp. 555-557, 1999.

[27] R. Allwinn, C. Schieferstein, S. Glauke, and H. W. Doerr, "Rapid diagnosis of primary dengue fever by the immunochromatographic test and by electron microscopy-a case report," Infection, vol. 27, no. 6, pp. 365-367, 1999.

[28] C. T. Sang, L. S. Hoon, A. Cuzzubbo, and P. Devine, "Clinical evaluation of a rapid immunochromatographic test for the diagnosis of dengue virus infection," Clinical and Diagnostic Laboratory Immunology, vol. 5, no. 3, pp. 407-409, 1998.

[29] D. W. Vaughn, A. Nisalak, S. Kalayanarooj et al., "Evaluation of a rapid immunochromatographic test for diagnosis of dengue virus infection," Journal of Clinical Microbiology, vol. 36, no. 1, pp. 234-238, 1998.

[30] S. D. Blacksell, J. A. Doust, P. N. Newton, S. J. Peacock, N. P. J. Day, and A. M. Dondorp, "A systematic review and metaanalysis of the diagnostic accuracy of rapid immunochromatographic assays for the detection of dengue virus IgM antibodies during acute infection," Transactions of the Royal Society of Tropical Medicine and Hygiene, vol. 100, no. 8, pp. 775-784, 2006.
[31] S. D. Blacksell, D. Bell, J. Kelley et al., "Prospective study to determine accuracy of rapid serological assays for diagnosis of acute dengue virus infection in Laos," Clinical and Vaccine Immunology, vol. 14, no. 11, pp. 1458-1464, 2007.

[32] T. T. T. Nga, K. T. D. Thai, L. P. Hoang et al., "Evaluation of two rapid immunochromatographic assays for diagnosis of dengue among Vietnamese febrile patients," Clinical and Vaccine Immunology, vol. 14, no. 6, pp. 799-801, 2007.

[33] M. Moorthy, S. Chandy, K. Selvaraj, and A. M. Abraham, "Evaluation of a rapid immunochromatographic device for the detection of IgM \& IgG antibodies to Dengue viruses (DENV) in a tertiary care hospital in South India," Indian Journal of Medical Microbiology, vol. 27, no. 3, pp. 254-256, 2009.

[34] Y. Y. Tan, S. D. Sekaran, S. M. Wang, F. Ahmed, A. Hossain, and B. K. Sil, "Development of ASSURE dengue IgA rapid test for the detection of anti-dengue IgA from dengue infected patients," Journal of Global Infectious Diseases, vol. 3, no. 3, pp. 233-240, 2011.

[35] F. Ahmed, H. Mursalin, M. T. Alam et al., "Evaluation of ASSURE Dengue IgA Rapid Test using dengue-positive and dengue-negative samples," Diagnostic Microbiology and Infectious Disease, vol. 68, no. 4, pp. 339-344, 2010.

[36] S. Alcon, A. Talarmin, M. Debruyne, A. Falconar, V. Deubel, and M. Flamand, "Enzyme-linked immunosorbent assay specific to dengue virus type 1 nonstructural protein NS1 reveals circulation of the antigen in the blood during the acute phase of disease in patients experiencing primary or secondary infections," Journal of Clinical Microbiology, vol. 40, no. 2, pp. 376-381, 2002.

[37] F. Najioullah, E. Combet, L. Paturel et al., "Prospective evaluation of nonstructural 1 enzyme-linked immunosorbent assay and rapid immunochromatographic tests to detect dengue virus in patients with acute febrile illness," Diagnostic Microbiology and Infectious Disease, vol. 69, no. 2, pp. 172-178, 2011.

[38] K. Y. Pok, Y. L. Lai, J. Sng, and L. C. Ng, "Evaluation of nonstructural 1 antigen assays for the diagnosis and surveillance of dengue in Singapore," Vector-Borne and Zoonotic Diseases, vol. 10, no. 10, pp. 1009-1016, 2010.

[39] V. Tricou, H. T. T. Vu, N. V. N. Quynh et al., "Comparison of two dengue NS1 rapid tests for sensitivity, specificity and relationship to viraemia and antibody responses," $B M C$ Infectious Diseases, vol. 10, p. 142, 2010.

[40] L. Osorio, M. Ramirez, A. Bonelo, L. A. Villar, and B. Parra, "Comparison of the diagnostic accuracy of commercial NS1based diagnostic tests for early dengue infection," Virology Journal, p. 361, 2010.

[41] V. T. Hang, N. M. Nguyet, D. T. Trung et al., "Diagnostic accuracy of NS1 ELISA and lateral flow rapid tests for dengue sensitivity, specificity and relationship to viraemia and antibody responses," PLoS Neglected Tropical Diseases, vol. 3, no. 1, p. e360, 2009.

[42] P. Y. Shu, C. F. Yang, J. F. Kao et al., "Application of the dengue virus NS1 antigen rapid test for on-site detection of imported dengue cases at airports," Clinical and Vaccine Immunology, vol. 16, no. 4, pp. 589-591, 2009.

[43] W. Chaiyaratana, A. Chuansumrit, V. Pongthanapisith, K. Tangnararatchakit, S. Lertwongrath, and S. Yoksan, "Evaluation of dengue nonstructural protein 1 antigen strip for the rapid diagnosis of patients with dengue infection.," Diagnostic Microbiology and Infectious Disease, vol. 64, no. 1, pp. 83-84, 2009. 
[44] P. Dussart, L. Petit, B. Labeau et al., "Evaluation of two new commercial tests for the diagnosis of acute dengue virus infection using NS1 antigen detection in human serum," PLoS Neglected Tropical Diseases, vol. 2, no. 8, p. e280, 2008.

[45] S. Zainah, A. H. A. Wahab, M. Mariam et al., "Performance of a commercial rapid dengue NS1 antigen immunochromatography test with reference to dengue NS1 antigen-capture ELISA," Journal of Virological Methods, vol. 155, no. 2, pp. 157$160,2009$.

[46] A. H. Ramirez, Z. Moros, G. Comach et al., "Evaluation of dengue NS1 antigen detection tests with acute sera from patients infected with dengue virus in Venezuela," Diagnostic Microbiology and Infectious Disease, vol. 65, no. 3, pp. 247-253, 2009.

[47] M. R. Q. Lima, R. M. R. Nogueira, H. G. Schatzmayr, and F. B. dos Santos, "Comparison of three commercially available dengue NS1 antigen capture assays for acute diagnosis of Dengue in Brazil," PLoS Neglected Tropical Diseases, vol. 4, no. 7, p. e738, 2010.

[48] S. D. Blacksell, M. P. Mammen, S. Thongpaseuth et al., "Evaluation of the Panbio dengue virus nonstructural 1 antigen detection and immunoglobulin $\mathrm{M}$ antibody enzyme-linked immunosorbent assays for the diagnosis of acute dengue infections in Laos," Diagnostic Microbiology and Infectious Disease, vol. 60, no. 1, pp. 43-49, 2008.

[49] V. Duong, S. Ly, P. Try et al., "Clinical and virological factors influencing the performance of a ns1 antigen-capture assay and potential use as a marker of dengue disease severity," PLoS Neglected Tropical Diseases, vol. 5, no. 7, p. e1244, 2011. 

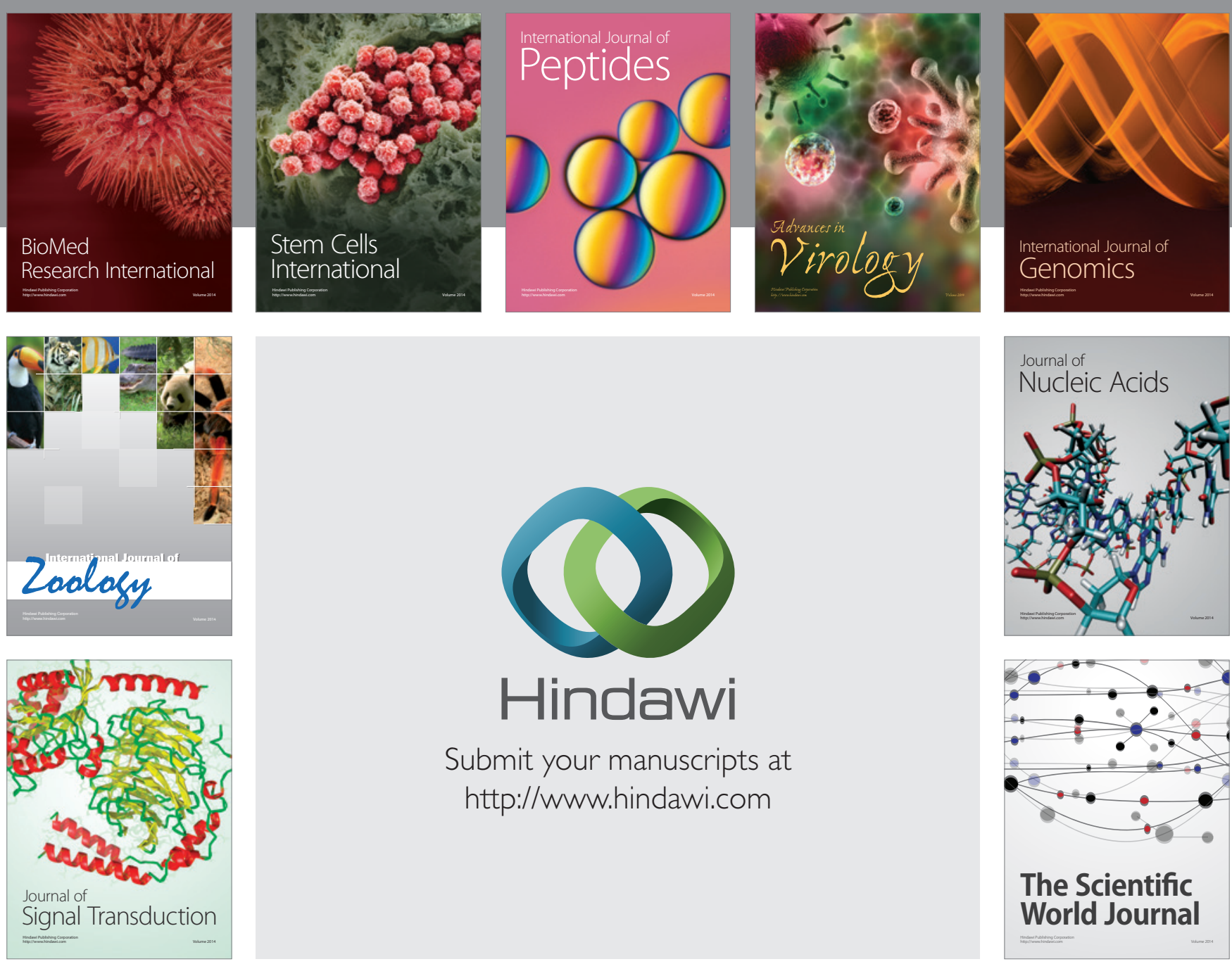

Submit your manuscripts at

http://www.hindawi.com
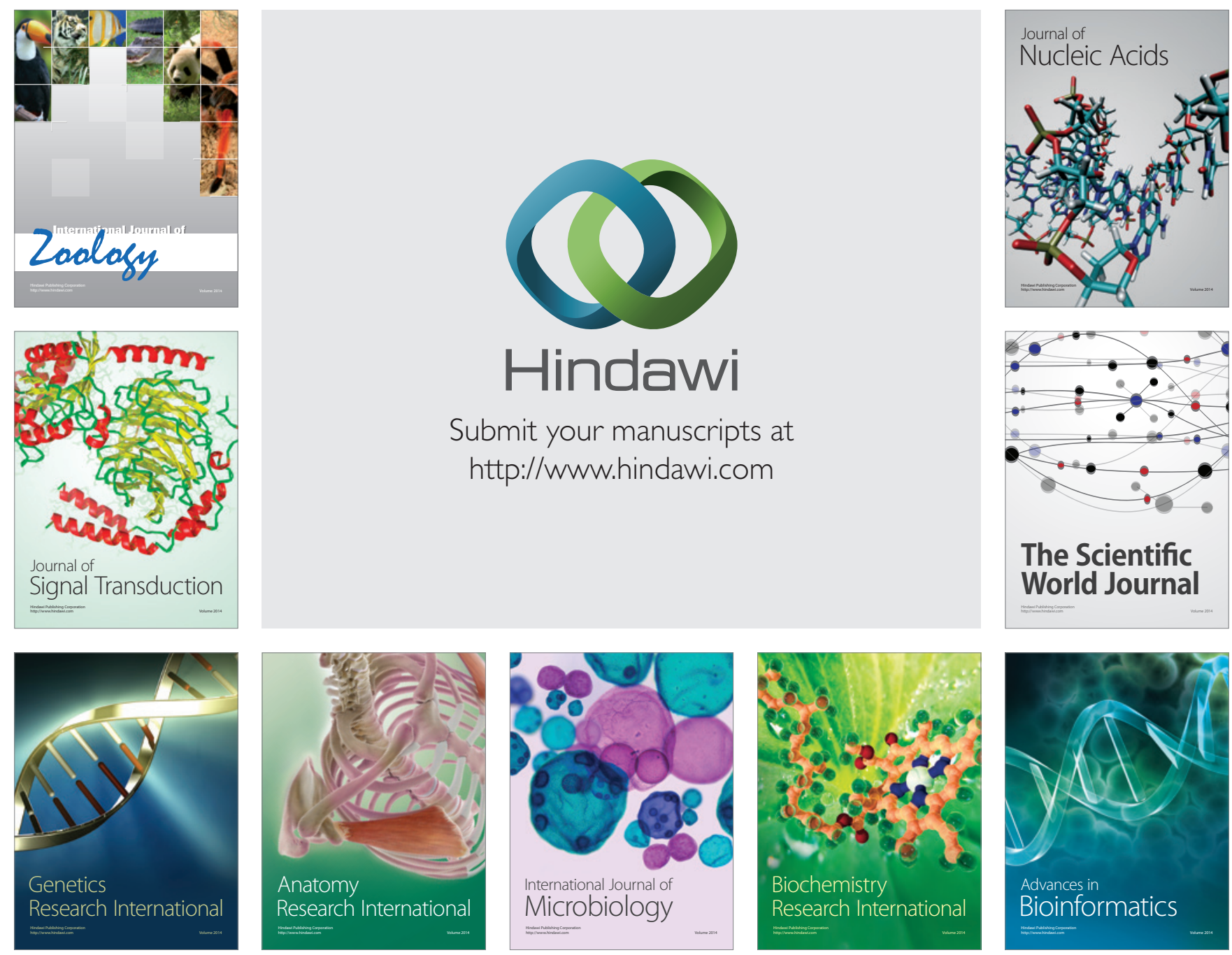

The Scientific World Journal
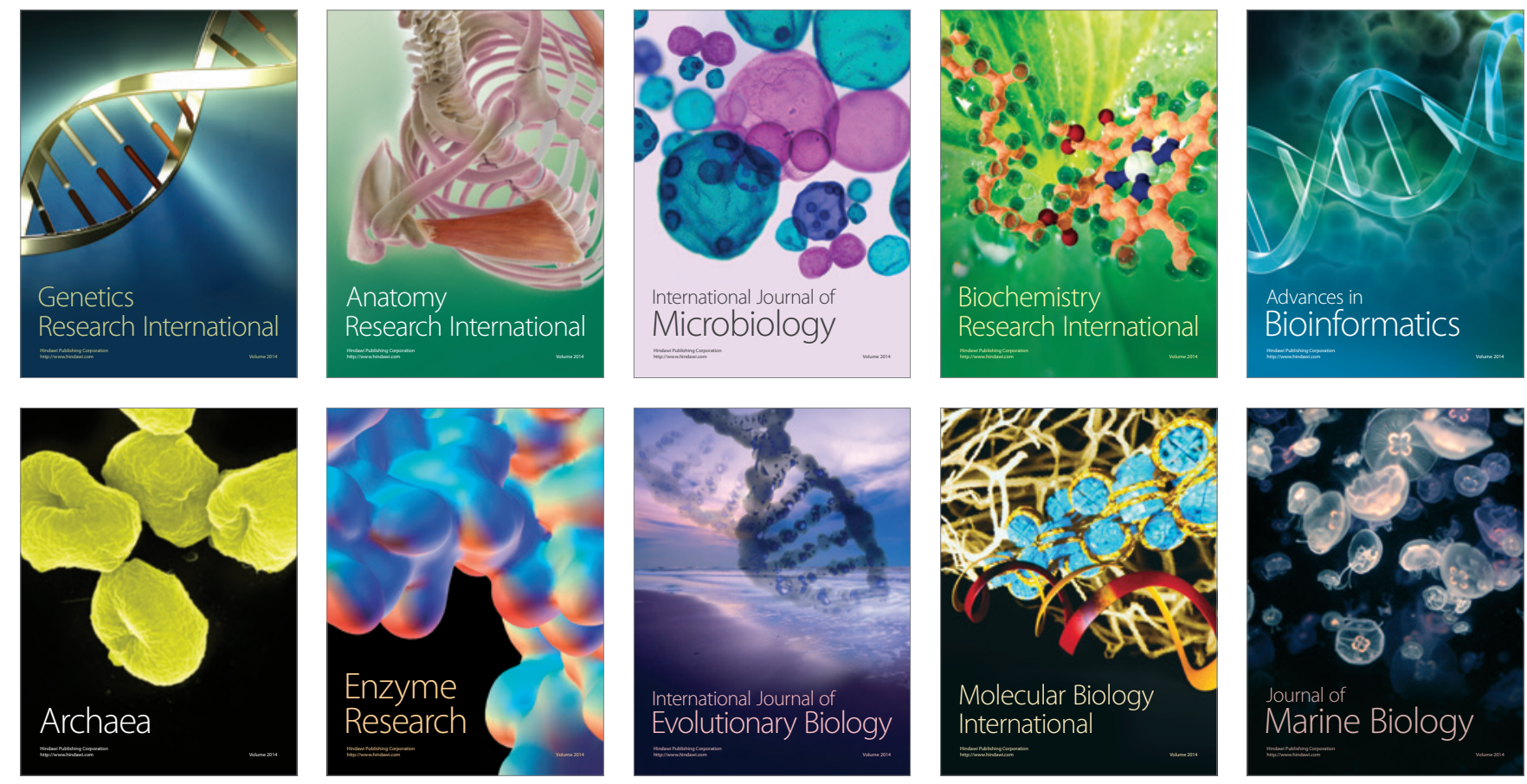